\title{
The public outreach programme of ENEAS
}

\author{
D.W. Kurtz ${ }^{1}$, K. Kolenberg ${ }^{2}$, Z. Kollath ${ }^{3}$, T. Teixeira ${ }^{4}$ \\ ${ }^{1}$ Centre for Astrophysics, University of Central Lancashire, Preston PR1 2HE, UK \\ 2 Institute for Astronomy, University of Vienna, Türkenschanzstrasse 17, A-1180, \\ Vienna, Austria \\ ${ }^{3}$ Konkoly Observatory, P.O. Box 67, H-1525 Budapest, Hungary \\ ${ }^{4}$ Department of Physics and Astronomy, Aarhus University, Ny Munkegade, 8000 \\ Aarhus C, Denmark
}

Everyone is fascinated with astronomy. Opportunities for astronomers to explain their work to the public with popular books, public talks, media interviews, television and radio programmes and internet sites are unlimited with very satisfying public response to all of our efforts. Recognising this, the directors of the European Network of Excellence in Asteroseismology, ENEAS, have created ENEAS-Outreach to help all of the more than 250 scientists in ENEAS bring the marvels of asteroseismology to the public. We, the four authors of this paper, are the board of directors of ENEAS-Outreach. Our purpose here is to call for input from members of the group, and to make available the outreach work of our colleagues for everyone's use. Public outreach is rewarding, but time-consuming. Our job as directors is to gather and create outreach material, and make that available to the entire group. It is not possible for only a few people who are interested in public outreach to give public talks, media interviews and presentations, and create internet sites in all the local languages of our member countries. What we in ENEAS-Outreach are doing is to create materials in our "connecting" language, English, that can then be adapted for local use all over Europe. These materials will be made available on the ENEAS website. To illustrate some of the possibilities, we explain here some our individual activities in public outreach.

\section{Don Kurtz:}

I have recently created a Powerpoint presentation for a public talk now called "Songs of the Stars: the real music of the spheres". Much of the material I have used has come from ENEAS members after a first public request by me. Fascinating presentations were sent to me by Mike Thompson, Yvonne Elsworth, Conny Aerts, Mario Monteiro, Katrien Kolenberg and Patricia Lampens. Sound 
files of stellar frequency spectra boosted into the audible range were provided by Zoltan Kollath, Gunter Houdek and Conny Aerts, and movies of non-radial pulsation modes were provided by Rich Townsend. It is clear that ENEAS has many members who are very active in public outreach and have already produced a rich source of material for the rest of ENEAS to use. Sylvie Vauclair has recently published a popular book, La Chanson du Soleil (The Song of the Sun), that has won the Orsay 2002 Scientific Book prize.

I had begun giving the talk "Songs of the Stars: the real music of the spheres" under the title "asteroseismology: the real music of the spheres" but quickly discovered that the big word "asteroseismology" in the title is scary to the public and cuts audience sizes. I have gathered material from internet sites, from members of ENEAS, and from other astronomers, as well as using my own to create this talk. Here is a press release written by Paula Opfer, a professional in public relations and marketing, from Australia where the talk was given in Perth, Melbourne and Sydney in conjunction with the IAU General Assembly in Sydney in July 2003. It is interesting and instructive for us scientists to see how PR people handle our subject.

A press release:

\section{Fancy a little night music?}

Did you know that the stars 'sing'? Professor Don Kurtz from the Centre for Astrophysics, University of Central Lancashire, UK, promises to let us in on the musical secrets of our Universe during his FREE talk. Held at 2 PM, Sunday, 20 July, 2003, at the Astro Expo, Exhibition Hall 5, of The Sydney Exhibition Centre at Darling Harbour, as part of The Australian Festival of Astronomy.

So Top 40 hits and dance tracks aren't doing it for you anymore? To hear the true sounds of the Universe, says Professor Don Kurtz, world-authority on asteroseismology, tune your ears a little higher. It seems that those stars so far above us are actually 'singing'.

Find that hard to believe?

During his free talk at Astro Expo on Sunday July 202003 at 2pm, Professor Kurtz will explain exactly how this heavenly symphony is composed. Beginning with its first listeners, the ancient Greeks...

The ancient Greeks believed that the planets and stars were embedded in crystal spheres that hummed as they spun around the heavens, making what they called 'the music of the spheres'. Pythagoras thought that the orbits of the planets had harmonic relationships. Unfortunately, Johannes Kepler was so enamoured of Pythagoras's idea that, in the early 1600s, he 
spent years trying to discover harmonic relationships among the planets, but ended up proving otherwise. The 'music of the spheres' was put on the backburner...

Then, in the 1970s, scientists began discovering that the sun and other stars actually do 'sing' - ringing from sound waves in them that cause them to vibrate, get hotter and cooler, brighter and dimmer, bigger and smaller, and change shape. These sound waves cannot get out of the star into the vacuum of space, so we do not 'hear' them directly. But scientists can detect the sounds that are there.

The detection process is what is now known as asteroseismology - the seismology (vibrations) of the stars. Using this method, scientists can 'look' beneath the surfaces of the stars, right into the unearthly maelstrom of the giant nuclear reactors that make up their cores.

Professor Kurtz promises to share the sounds of the stars (including an amazing group of the strangest stars in the sky, discovered by Kurtz himself) with his audience.

Britney Spears, eat your heart out.

\section{Don Kurtz:}

With ENEAS-Outreach all members of ENEAS are free to use the material for this presentation, to adapt it to be given in their own languages, to personalise it for their own research specialty, and to provide feedback to ENEAS-Outreach so that we can continue to improve and increase the material available to us all. Some of the very best material has been produced by Zoltan Kollath. I find his "Cepheid Horn" particularly entrances audiences.

\section{Zoltan Kollath:}

Parallels in sciences and the arts can help a lot to demonstrate the nature of physical processes in stars for a general public. Mathematical and physical similarities between pulsating stars and musical instruments have been discussed by Buchler et al. (1997). A "Cepheid Horn" they developed has the overtone response of a Cepheid, rather than the purely harmonic response of a musical instrument and can be used to show the overtone structure of stellar oscillations. I have created several animations to illustrate the similarities of musical instruments and stellar physics that will be available on the ENEAS web site, and can be found in DWK's "Songs of the Stars: the real music of the spheres". I have created an electronic music file of Bach's Choral prelude "Ich ruf' zu dir, Herr Jesu Christ" as it sounds with the overtone response of the Cepheid Horn. 
It is slightly dissonant, as is to be expected, but very pleasing to listen to. Audiences are captivated by it. It is accompanied by an animation of a pulsating Cepheid with the Cepheid horn rotating to be seen from different aspects.

My animations are used for many different audiences: from school children to physics teachers and amateur astronomers; they have also appeared in television science programmes in Hungary. Virtual musical instruments based on the physics of the stars and observations can be used to orchestrate any piece of music - with that we reach the borderline of science and art. You can talk about physics and at the same time entertain the audience. Such musical experiments were aired in a radio broadcast and were used as a background music for a TV programme on Konkoly Observatory. The other way around is true, too: Astronomers are as fascinated by this science/art boundary as is the public.

A 5-minute-long animation titled "The Music of the Stars" was on continuous display for more than a year at the "Dreamers of Dreams: Hungarians of World Renown" exhibition at the "Millenŕis Park Exhibition and Events Center" complex that was built during $2000 / 2001$ to commemorate the $1000^{\text {th }}$ anniversary of the founding of the Hungarian state. During the August 2003 JENAM meeting I will give a public talk entitled "The Music of Cosmos".

My animations, sound files and astro-musical creations will be made available to ENEAS members for public outreach all across Europe.

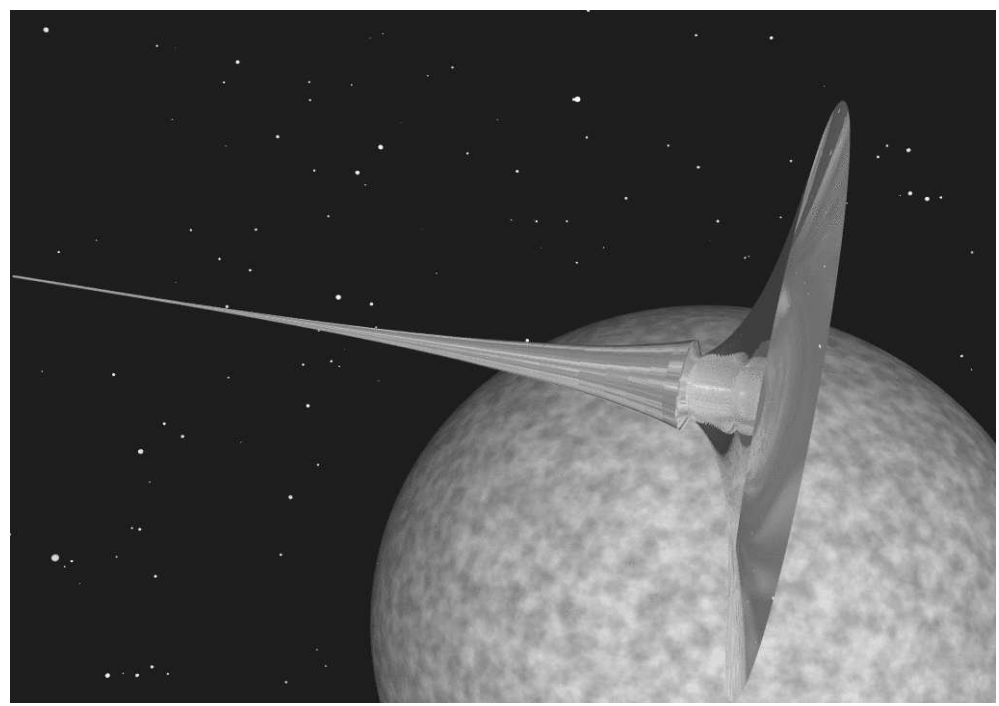

Figure 1: The Cepheid Horn 
Katrien Kolenberg:

Often when people ask me what my work is about, I use the metaphor of a peeled mandarin, to illustrate how one can imagine nonradial sectoral pulsation. A rainbow, a passing ambulance, a stone thrown into water ... there are enough phenomena in daily life that can be directly related to the work we are doing, and can be used to make it clear to whomever is interested, adults or children. And sometimes a little drawing also helps.

I have been interested in illustrating astronomy through art since my teenage years as an amateur astronomer. After my studies, my popularisation activities focused more and more on stellar astronomy. Just like some of my colleagues, I gave lectures for the interested public. My predilection for handmade things was enjoyed to the full when designing poster and cover illustrations for some conferences and symposia, e.g., for IAU Symposium 191, IAU Coll. 176, the 53rd Dutch Astronomical Conference, and several posters for events organized by the local amateur astronomy group. When some of my colleagues in Louvain finished their PhDs, I was asked to design the covers for their theses, displaying an "artistic and colourful impression" of the work they were presenting. I created the logo for the Institute for Astronomy of Louvain (Belgium) and, finally, designed the logo for ENEAS that can be seen on this report and all ENEAS documents.

For ENEAS-outreach my presentations are available for others to use the artwork (with acknowledgement). Time permitting, on special request I am prepared to liven up astronomers' presentations by designing, e.g., an appropriate background for a PowerPoint presentation. I can also design logos and illustrations for posters and covers. I hope that from this example we will find amongst ENEAS members others with creative hobbies that can make our science more accessible to the public and give greater pleasure to everyone, astronomers included!

\section{Teresa Teixeira:}

The asteroseismology group at the Department of Physics and Astronomy of the Aarhus University (Denmark) has for the past year been involved in a range of public outreach activities, many of them involving schools. Because of the specificity of the main subject of our research, asteroseismology, our outreach and education activities do not solely concentrate on that subject, but rather include it in a more general background of Stellar Astrophysics.

1. Activities with and for schools

(i) We have organized visits of 1st-year high-school classes (16-year-olds) to spend a day at the department. The students are introduced to the subject of the Sun, solar structure and activity in a informal lecture given by one of the 
researchers in the group. After the lecture, the students have the opportunity to work on computer-based exercises prepared by members of our group, using typically data from satellites (e.g. SoHO). To work on these exercises they are assisted by members of the group and/or $\mathrm{PhD}$ students. That allows them to ask and answer questions from researchers, and to challenge and to be challenged! During the visit, and weather allowing, the students are also given the opportunity to make their own continuum and $\mathrm{H}-\alpha$ observations of the Sun.

(ii) In collaboration with high-school teachers, we will now be working on preparing more advanced exercises using astronomical observations to illustrate concepts taught in physics in the 2nd and 3rd years in high-school (17- to 19year-olds). Some of the techniques and concepts from asteroseismology will be used in such exercises.

(iii) We have hosted visits of individual students from the basic school (13to 15-year-olds), where they have the opportunity to spend two or three days with one of the researchers in the group, learning about the research carried out in the group through discussions and relevant exercises.

2. Activities for the public in general.

(i) Based on the Rømer/MONS project, we produced a short planetarium presentation for journalists and decision makers. Following the success of that presentation, we are now preparing an educational planetarium presentation aimed at high-school level and the public in general. The main topics of that presentation will be stellar structure and asteroseismology, and the study of extrasolar planets, using as "anchor" points three space missions: SoHO, Eddington and Darwin. We will also prepare supporting educational material as a complement to the planetarium session, to be used in connection with school visits and public talks.

(ii) Talks at observatories and astronomy clubs, which are often attended by families.

3. Other activities.

Following reactions to press releases on asteroseismology, we have also provided sound material to a composer and to a music company who were interested in the sounds of stars for artistic purposes.

Preparing and carrying out all these activities is extremely demanding and time-consuming, but also extremely rewarding. We have learned a lot about people's interests and expectations, about perceptions and misconceptions, and also about our successes and (many) shortcomings at presenting our subject of research. But all reactions have been extremely positive and encouraging. At the end of the day we will feel we have been successful if our (different) audiences go home feeling they have learned something new, if they will have felt some of the excitement that drives us in research, if they feel they can understand the principles behind it, if they have seen some beauty in astronomy, 
in pictures, in sounds, in words, or if, like someone one day told me, if we make them dream. Then we will have reached out to our public.

All of us:

Many ENEAS members are already very active in public outreach. They know the pleasure of presenting astronomy to the public, and they know the rewards. Others who have thought about this, but have not yet become involved should think about how they, themselves might benefit. We all know how science benefits from public understanding and enthusiasm for our work, but you, the astronomer, can benefit, too. Of course, many of you are teachers and enjoy being on stage with a captive audience. The general public has a wide age range and wide background knowledge from experts to beginners, but people who attend your public lectures are enthusiastic. Their applause after a presentation is not the polite brief applause of a professional audience to a colloquium or meeting talk; it is laud, sustained, full of the pleasure they have received from your presentation - sometimes a bit embarrassingly so. Try it - you'll like it. You can speak at schools, science festivals, to business people, to clubs ... even on cruise ships! Most of this is all-expenses-paid, frequently for you and your partner. Once you start this you will find that a talk or two can pay for a luxury holiday. You can "sing for your supper".

ENEAS-Outreach will provide material you can adapt from English to your own language. We hope that talks polished by the work and knowledge of many ENEAS members will be presented to audiences all over Europe. We also request that you share your own material with ENEAS members though the ENEAS website. We intend that the ENEAS-Outreach part of the website will be developed to include the presentation material used for public talks. It will be developed into forms useful for schoolchildren for learning astronomy, as well as anyone searching the web for asteroseismology. With the phrase "songs of the stars" we will get some surprised visitors to our website. Once they see what is there, many of them will stay for a while to find out about asteroseismology.

Acknowledgments. We thank Paula Opfer, of The Axis Public Relations \& Marketing Company, Sydney, Australia, for permission to use the press release "Fancy a little night music".

\section{References}

Buchler, J. R., Yecko, P.E., Kollath, Z. 1997, A\&A 326, 669 